\title{
Two Training-Load Paradoxes: Can We Work Harder and Smarter, Can Physical Preparation and Medical Be Teammates?
}

\author{
Tim J. Gabbett and Rod Whiteley
}

\begin{abstract}
The authors have observed that in professional sporting organizations the staff responsible for physical preparation and medical care typically practice in relative isolation and display tension as regards their attitudes toward training-load prescription (much more and much less training, respectively). Recent evidence shows that relatively high chronic training loads, when they are appropriately reached, are associated with reduced injury risk and better performance. Understanding this link between performance and training loads removes this tension but requires a better understanding of the relationship between the acute:chronic workload ratio (ACWR) and its association with performance and injury. However, there remain many questions in the area of ACWR, and we are likely at an early stage of our understanding of these parameters and their interrelationships. This opinion paper explores these themes and makes recommendations for improving performance through better synergies in support-staff approaches. Furthermore, aspects of the ACWR that remain to be clarified-the role of shared decision making, risk:benefit estimation, and clearer accountability—are discussed.
\end{abstract}

Keywords: shared decision making, acute:chronic workload ratio, injury, performance

Training loads determine physical performance ability, albeit in a complex nonlinear manner, subject to individual variations in response. In large, it is suggested that physically appropriate, higher training loads are associated with more positive performance adaptations up to some point - this point being the creation of an overuse injury. Indeed it has recently been suggested that an overuse injury is, by definition, a training load prescription error. ${ }^{1}$ Given that overuse injury can form the great majority of injury seen in some sports, it could be argued that the majority of injury burden is driven by incorrect training practice. Viewed through this simplistic prism, in the context of many professional sporting environments, the physical preparation and medical staff are at odds advocating higher and lower training loads respectively. This superficial understanding of the relationships between training load, injury, and performance have led to a dichotomizing of approaches wherein if low injury rates are the primary aim, training loads should be minimized and alternately training loads should be maximized where physical preparation is the principal driver.

More recently a model has been developed suggesting that the absolute training load at any point in time is a less useful predictor of injury than considering the individual's current training load, and training load history. Initially it was observed that the week-on-week changes in training load are more predictive of injury risk than the absolute training load at any given point in time ${ }^{2}$ questioning the more simplistic approaches outlined above.

Subsequently Banister's suggested performance model investigating the balance between "fitness" (chronic training load, typically 4-wk rolling average) and "fatigue" (acute training load, typically 1 $w \mathrm{k})^{3}$ has proven a useful starting point for examination of training injury rates. The acute:chronic workload ratio (ACWR), an index

Gabbett is the Inst for Resilient Regions, University of Southern Queensland, Ipswich, Australia. Whiteley is with Aspetar Orthopedic and Sports Medicine Hospital, Doha, Qatar. Address author correspondence to Rod Whiteley at Rodney.whiteley@ aspetar.com. of the workload performed, relative to the workload the athlete has been prepared for, is more strongly associated with injury rates than absolute workload in isolation. ${ }^{4,5}$

This information has been synthesized into a working model ${ }^{2}$ wherein the ultimate aim is higher absolute training loads (as these will confer the greatest performance gains) being achieved through staged increases in weekly training loads. Specifically it is suggested that weekly increases in loading be capped, depending on the appetite for risk of injury, at somewhere between $10 \%$ and $50 \% .^{2}$

\section{By Definition, Preparing for the Average Demands Means Underpreparation Half of the Time.}

The aim of physical preparation is to adequately prepare the athlete for the demands of competition. Accordingly a knowledge of these demands is critical for accurate prescription. We suggest that some errors may have been inadvertently introduced where athletes have been prepared for the average match demands. By definition, if an athlete is only capable of tolerating the average demands of a match, then they are underprepared for half of the competition they will endure. Accordingly we suggest that a key aim of preparation is to ready an athlete for any match demands within reason. Clearly these will be higher than average demands, and are typically higher than usual training loads. ${ }^{6}$ By considering these aims in the context of safe weekly increases in loads, athletes can scale these heights through safe steps while accruing higher chronic training loads. Such higher training loads appear to also be associated with lower injury levels likely through higher performance ability rendering typical match demands as being relatively lower for any given individual. This protective effect of high training loads has recently been documented in the particularly problematic situation of periods of relative match congestion. ${ }^{7}$ 


\section{We Need to Interlink Those Responsible for Training Loads, Injury, and Performance.}

Currently, those responsible for performance, preparation, and medical care typically work in silos despite routinely paying lip service to such terms as multidisciplinary and athlete-centered. Coupled with the commonly held inappropriately simplistic beliefs surrounding training load and injury outlined earlier, we believe that this has resulted in these different, apparent teammates (coach, preparation, and medical staff), working as competitors who have different motivations (win games, have the largest training volumes, have the lowest injury rates, respectively) who attempt to reach these goals by typically different means (any means necessary, more training, less training, respectively).

\section{Consider Match Performance as a Primary Goal, and This Competition Disappears.}

If both the weight of focus of a program is primarily on injury prevention (as opposed to performance) and high loads are equated with injury, then players will constantly be managed away from their threshold. Certainly this will result in never pushing players to a level that will result in increased injury risk, but equally they will never develop physical qualities which allow optimal performance. ${ }^{2}$
Similarly, when the focus is purely on filling every hour of every day with heavy training loads irrespective of the athlete's loading and injury history, then the inevitable consequence is injury, and performance and coaching staff are managing at least some of their athletes away from performance via injury (Figure 1). On-field performance clearly suffers in the short and long term when players are unable to complete their planned training. ${ }^{8}$ Once the nexus between appropriate loading-with the goal of ultimately high chronic loads - and performance is understood within a program, the competition between preparation and medical disappears, and a truly cohesive athlete-centered approach can be realized. However, the converse is seen much more commonly in practice whenever these 2 team members fail to appreciate these links, and each member of the team pulls the athlete in opposite directions.

When preparation staff optimize training loads-we suggest via appropriate use of the ACWR - to create high chronic training loads in their athletes, well in excess of the average demands of competition, performance will be maximized, and injury minimized. When each of the members of the support staff understands and adopts this approach to their care, the conflict outlined earlier disappears. Alternately if considered through the prism of "what would make my athletes' performance improve?" then each member should similarly arrive at the conclusion that appropriately high chronic training loads achieved through staged monitored increases will be the answer.

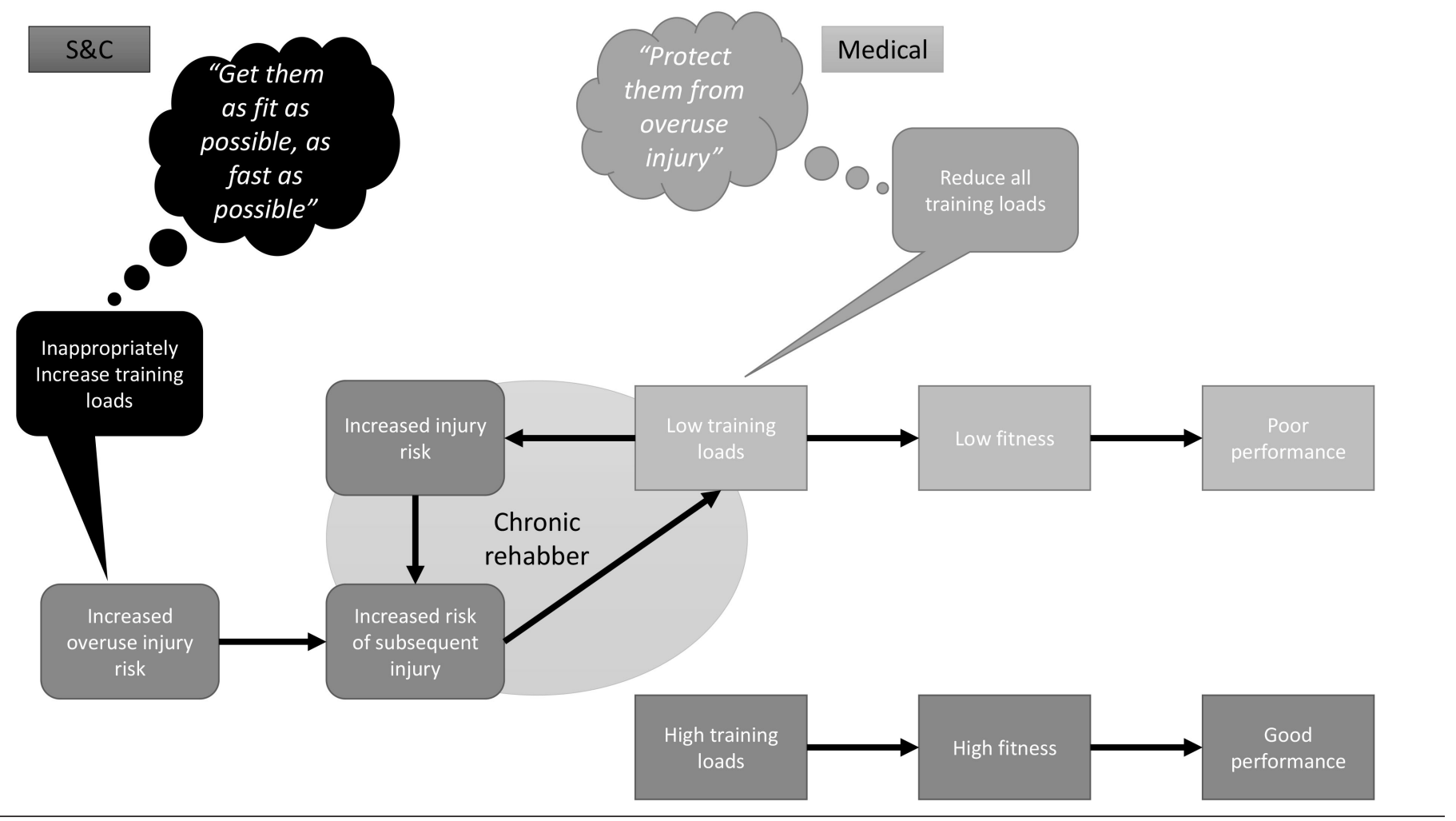

Figure 1 - Depiction of how an inadequate understanding of the links between training loads, injury risk, and performance can inadvertently give rise to increased injury risk and the "chronic rehabber" in a team. By forcing low training loads on a player, either deliberately through a policy of "protecting" from overuse injury through reduced training load or indirectly when an overuse injury forces a reduction in training load, the player gets into a cycle where this reduced training load increases injury risk when the player is exposed to game loads, or "normal" training loads. Typically, medical staff are at fault for incorrectly reducing training loads, and physical preparation staff are at fault for inappropriately working players too hard when incomplete understanding of the "train smarter and harder" approach is present. 


\section{The Athlete's Role in Training Loads Must Be Considered, and Athletes Must Be Accountable.}

Athletes rightly take credit for great performances, and will bear the brunt of perceived failure in competition. We believe that this accountability should be extended beyond competition periods. Some sports (eg, American football, Premier League soccer) mandate that athletes have extended periods away from formal training with no supervision of workloads permissible by the organizations. If athletes fail to understand the relationships between periods of reduced activity and the associated reductions in chronic loading that creates elevated injury risk, then it is understandable that they would take this opportunity to "rest and recuperate." If their primary aim is maximizing performance and minimizing injury risk, then a better understanding of the relation between training loads, injury, and performance will ensure that athletes independently continue their preparation during these "down times." It is incumbent on those with knowledge of safe training practices to inform the athletes of this, and to provide athletes with suggestions for continued training. Athletes should also be aware that they should be held accountable for any subsequent poor performance or injury that can be directly traced to inadequate preparation in which they have knowingly contributed.

\section{Your Sport Still Needs to Describe the Correct Training and Performance Metrics.}

To this point we have deliberately used the generic descriptors of "appropriate training loads." What constitutes the exact parameters of these training loads, and how are they best measured? Here again we likely have much to discover.

Despite both athletes being required to run and throw, high speed running meters is a meaningless metric for baseball pitchers, as is total throws for a football goalkeeper. Appropriate metrics will be associated with performance as well as injury risk. In all likelihood, the optimal measures for each sport, and perhaps each individual, have not yet been described.

It needs to be recalled that the demands of the match will be specific to individual tissues and energy systems for any given athlete's role within any given sport. The rate-limiting tissue and/or energy systems need to be targeted first and foremost both during preparation and monitoring. High chronic training loads on an exercise bike are not the same as high chronic overground sprinting loads as they will induce different changes in the musculature of the thigh. If the ultimate aim of the sport is repeated-sprint ability, then accrual of high chronic cycling loads (at the expense of high-speed running) are likely detrimental to performance and injury risk.

We are at an early stage in our understanding of the specifics of the relationships between training load, performance, and injury risk, and many questions remain incompletely answered. The following is a limited set of such questions and our current thinking on these.

\section{Why 1 Week:4 Weeks for the Acute: Chronic Workload Ratio?}

We do not know if different acute and chronic workload timing parameters will give better results, and we need to explore this further. Current work has largely been limited to describing associa- tions between training load and observed injury. Future work needs to validate these approaches in larger data sets to establish their predictive veracity. Speculatively in sports that require significant physiological adaptation to determine performance, (eg, triathlon, marathon, road cycling) a longer chronic duration may be appropriate, whereas competitions with congested fixture schedules (eg, National Basketball Association, English Premier League) may use shorter acute loading periods.

\section{What About Different Tissues and Their Response to Load (Time Course)?}

We know that different tissues have different responses to different loading, with different time courses for adaptation and remodeling. For example, the repeated bout effect suggests that one or two relatively high-load and-volume sessions of eccentric exercise may protect individuals from eccentric induced delayed-onset muscle soreness for up to 6 months (in the same muscle group performing the exercise). ${ }^{9}$ Tendons, however, are seen to respond acutely to high volumes of running with variation in their creation and destruction of collagen and associated building blocks across a relatively short time period (days) with differences in the time course for creation and turnover of collagen and response to a period of training. ${ }^{10,11}$ This may have implications for upper limits of loading in terms of time between exercise insults that can be safely tolerated. Speculatively we can use this information to our advantage to optimize positive adaptation of tissue through loading, however much more work needs to be done to describe all the parameters surrounding this information.

\section{What About Other Modifiers of Response Such as Athlete Age and Different Body Tissues?}

Most likely an athlete's age is an important influencing factor on their response to training, and its ability to both enhance performance and mitigate injury risk. For example, recent data suggests that younger players are more resistant to injury when having adverse strength and architectural properties of the hamstring muscles in the context of prevention of hamstring injury. ${ }^{12}$ Similarly an athlete's anabolic response to hypertrophy training varies across their lifespan ${ }^{13}$ which should alter an individual athlete's ability to adapt positively to loading.

Adolescent athletes are susceptible to certain injuries at their maturing physeal (cartilaginous) growth plates ${ }^{14}$ and require different approaches to loading. In particular it would seem that these growth plates respond differently to compressive and tensile forces, ${ }^{15}$ and it should be recalled that in many activities, a single growth plate may be under both types of load in different parts.

These different growth plates mature at different ages. For example the calcaneal physis in the heel is seen to mature earlier than the tibial physis at the knee, and accordingly the peak incidence of Sever's disease ${ }^{16}$ of the calcaneal apophysis is younger than that of Osgood-Schlatter's disease ${ }^{17}$ of the knee. It should be recalled that the different growth plates mature from as early as 10 (calcaneal apophysis in the heel) to as late as 25 years old (pubic apophysis in the pelvis/groin ${ }^{18}$ ) and that there is marked interindividual variability in this.

Finally, different tissues and structures are seen to be more at risk across an athlete's life span. Based on the results from cricket, ${ }^{19}$ it is likely that different tissue types will respond differently to different loads. For example, bone stress and tendon injuries have been shown to increase in response to high acute workloads, 
while joint injuries occur in response to high career workloads. Conversely, higher chronic workloads have been shown to protect against muscle injuries.

\section{Is Session RPE Less Useful Than a GPS Metric?}

Notwithstanding discussions regarding the differences between planned and actual training loads, session RPE is most likely the simplest loading metric to apply to athletes across team and individual sports. Increasingly advanced technological solutions are becoming available at an astonishing rate, with many claims being made regarding the veracity of standard and novel measurements. When considering the purpose of these metrics, we need to return to the primary aims of describing appropriate loads to maximize physical improvement while simultaneously minimizing injury risk. In many situations (eg, in subelite populations), it would appear that the added accuracy provided by the more complex measures are clinically insignificant, and we would suggest not worth the additional investments. As technology continues to improve, this statement will almost certainly need revision, but we would suggest that before investing in expensive technology, performance and medical staff should consider the demands of the sport, and whether the technology can measure the critical "metrics" for the sport in question.

\section{What About "Shock Blocks"?}

While we suggest that steady careful increases in load provides the optimal method of shifting an athlete toward high chronic training loads, occasionally logistical or other demands may call for brief periods of markedly elevated loading. We suggest that there could be some very useful physical and psychological gains that can be had through a few days of very heavy training at the end of a very good preseason program, but almost certainly not the week before a final. The difficulty then lies not in the ability to program very high intensity workloads, but to accurately know when these are appropriate, and this means a careful consideration of the risks and benefits associated with these approaches with all stakeholders before embarking on this strategy.

Equally important is the opposite to a shock block and knowing when it is suitable to temporarily drop training loads. Examples would include identification of signs of nonfunctional overreaching $^{20}$ or tapering before an important event. ${ }^{21}$

\section{Training, and Changing Training, Is Not Risk-Free-Risk-Benefit Needs to Be Considered at All Stages.}

Understanding training loads involves embracing the uncertainty associated with these risks and rewards. An event that is $90 \%$ likely to happen will not happen $10 \%$ of the time. Considering this risk in its context, as well as communicating and discussing these risks is key to truly having an athlete-centered program. Attempting to quantify the risk is the important first step, and here we suggest that understanding the ACWR is central. Equally important is then understanding all the associated risk modifiers which are just as crucial in making a final decision regarding loading. Consider an example where 2 athletes have known risk of serious injury of 5\% and $95 \%$ respectively, if they play in a game. Cursory examination would suggest that we would be more likely to subject the player with the lower risk to loading (eg, a match). Consider now the fol- lowing modifiers: The low-risk athlete is 19 years old and considered as the likely future captain of the national team, and the loading we are considering is a preseason intrasquad friendly. Our high-risk player, by contrast, is aiming to play the final professional game of his career, the national league final, at 34. The coaching staff believe that the only chance of victory in this game is if he plays, and there is the chance that if he plays successfully, he may have his career extended with another year's contract in a foreign country. We would argue that the young player should almost definitely not play, while the veteran almost certainly should. We would surely argue that these factors should be considered by all parties before making the decisions to play or not, and carry the consequences equally among all decision makers.

Quantifying risks and benefits presents 1 further difficulty. It is practically impossible to count injuries that did not happen, and poor performances that did not occur. The approach required is minimizing risk, and in doing so, this necessarily involves quantifying the apparent risk (injury, poor performance) in terms of the training loads, and then sharing the decisions about these risks with all stakeholders (coach, strength and conditioning, medical, athlete, manager). Clear lines and decision trees need to be established a priori, with appropriate mechanisms in place to ensure these are being adhered to once agreed upon. These procedures should be reviewed in light of their apparent success or failure after the fact, and adjusted accordingly in an iterative process.

\section{How Does Your System Hold Up Under This Scrutiny?}

An entire system, including all its components needs to be considered as an organic whole. From the micro level of the athlete "putting in" over the last $5 \mathrm{~m}$ of the last repetition of a session of repeated 400-m sprints to the macro level of a sport which enforces a break from training and outlaws any monitoring of athlete behavior during this time, and everything in between. Each of these factors is contributing to wins and losses, and to a certain extent injuries, to a differing degree, and represents opportunities for global improvement in a program.

\section{References}

1. Drew MK, Purdam C. Time to bin the term 'overuse' injury: is 'training load error' a more accurate term? Br J Sports Med. 2016; doi:10.1136/ bjsports-2015-095543.

2. Gabbett TJ. The training-injury prevention paradox: should athletes be training smarter and harder? Br J Sports Med. 2016;50:273-280 doi:10.1136/bjsports-2015-095788. PubMed

3. Banister EW, Calvert TW, Savage MV, Bach T. A systems model of training for athletic performance. Austr J Sports Med. 1975;7:57-61.

4. Hulin BT, Gabbett TJ, Blanch P, et al. Spikes in acute workload are associated with increased injury risk in elite cricket fast bowlers. $\mathrm{Br}$ J Sports Med. 2014;48:708-712 doi:10.1136/bjsports-2013-092524. PubMed

5. Hulin BT, Gabbett TJ, Lawson DW, Caputi P, Sampson JA. The acute:chronic workload ratio predicts injury: high chronic workload may decrease injury risk in elite rugby league players. Br J Sports Med. 2016;50:231-236 doi:10.1136/bjsports-2015-094817. PubMed

6. Gabbett TJ, Jenkins DG, Abernethy B. Physical demands of professional rugby league training and competition using microtechnology. J Sci Med Sport. 2012;15:80-86 doi:10.1016/j.jsams.2011.07.004. PubMed 
7. Hulin BT, Gabbett TJ, Caputi P, Lawson DW, Sampson JA. Low chronic workload and the acute:chronic workload ratio are more predictive of injury than between-match recovery time: a two-season prospective cohort study in elite rugby league players. $\mathrm{Br}$ J Sports Med. 2016; 10.1136/bjsports-2015-095364. PubMed

8. Raysmith BP, Drew MK. Performance success or failure is influenced by weeks lost to injury and illness in elite Australian track and field athletes: a 5-year prospective study. J Sci Med Sport. 2016; doi:10.1016/j. jsams.2015.12.515. PubMed

9. Nosaka K, Sakamoto K, Newton M, Sacco P. How long does the protective effect on eccentric exercise-induced muscle damage last? Med Sci Sports Exerc. 2001;33:1490-1495. PubMed doi:10.1097/ 00005768-200109000-00011

10. Langberg H, Rosendal L, Kjaer M. Training-induced changes in peritendinous type I collagen turnover determined by microdialysis in humans. J Physiol. 2001;534:297-302. PubMed doi:10.1111/j.1469-7793.2001.00297.x

11. Langberg H, Skovgaard D, Petersen LJ, Bulow J, Kjaer M. Type I collagen synthesis and degradation in peritendinous tissue after exercise determined by microdialysis in humans. J Physiol. 1999;521(Pt 1):299-306. PubMed doi:10.1111/j.1469-7793.1999.00299.x

12. Timmins RG, Shield AJ, Williams MD, Lorenzen C, Opar DA. Architectural adaptations of muscle to training and injury: a narrative review outlining the contributions by fascicle length, pennation angle and muscle thickness. Br J Sports Med. 2016; doi:10.1136/ bjsports-2015-094881. PubMed

13. Walker S, Santolamazza F, Kraemer W, Hakkinen K. Effects of prolonged hypertrophic resistance training on acute endocrine responses in young and older men. J Aging Phys Act. 2015;23:230-236 doi:10.1123/japa.2013-0029. PubMed
14. Sailly M, Whiteley R, Johnson A. Doppler ultrasound and tibial tuberosity maturation status predicts pain in adolescent male athletes with Osgood-Schlatter's disease: a case series with comparison group and clinical interpretation. Br J Sports Med. 2013;47:93-97 doi:10.1136/ bjsports-2012-091471. PubMed

15. Stokes IA, Clark KC, Farnum CE, Aronsson DD. Alterations in the growth plate associated with growth modulation by sustained compression or distraction. Bone. 2007;41:197-205 doi:10.1016/j. bone.2007.04.180. PubMed

16. Scharfbillig RW, Jones S, Scutter SD. Sever's disease: what does the literature really tell us? J Am Podiatr Med Assoc. 2008;98:212-223. PubMed doi:10.7547/0980212

17. Krause BL, Williams JP, Catterall A. Natural history of OsgoodSchlatter disease. J Pediatr Orthop. 1990;10:65-68. PubMed doi:10.1097/01241398-199001000-00012

18. Sailly M, Whiteley R, Read JW, et al. Pubic apophysitis: a previously undescribed clinical entity of groin pain in athletes. Br J Sports Med. 2015;49:828-834 doi:10.1136/bjsports-2014-094436. PubMed

19. Orchard JW, Blanch P, Paoloni J, et al. Cricket fast bowling workload patterns as risk factors for tendon, muscle, bone and joint injuries. $\mathrm{Br}$ J Sports Med. 2015;49:1064-1068 doi:10.1136/bjsports-2014-093683. PubMed

20. Schmikli SL, Brink MS, de Vries WR, Backx FJ. Can we detect nonfunctional overreaching in young elite soccer players and middle-long distance runners using field performance tests? Br J Sports Med. 2011;45:631-636 doi:10.1136/bjsm.2009.067462. PubMed

21. Mujika I, Goya A, Ruiz E, et al. Physiological and performance responses to a 6-day taper in middle-distance runners: influence of training frequency. Int J Sports Med. 2002;23:367-373 doi:10.1055/ s-2002-33146. PubMed 